gle-step method of RNA isolation by acid guanidinium thiocyanate-phenol-chlorophorm extraction. Anal. Biochem. 162:156-159.

2.Nevins, J.R. 1987. Isolation and analysis of nuclear RNA. Methods Enzymol. 152:234241.

3.Vareli, K., M. Frangou-Lazaridis and $\mathbf{O}$. Tsolas. 1995. Prothymosin alpha mRNA levels vary with c-myc expression during tissue proliferation, viral infection and heat shock. FEBS Lett. 371:337-340.

This work was supported by Grants PENED 346/89 and 677/91 from the Greek Secretariat of Research and Technology to M. F-L. Address correspondence to Maria Frangou-Lazaridis, Laboratory of Biological Chemistry, University of Ioannina Medical School, 45110 Ioannina, Greece.

Received 19 June 1995; accepted 25 January 1996.

Katerina Vareli and Maria Frangou-Lazaridis University of Ioannina Medical School

Ioannina, Greece

\section{Pouring Gradients Using a Cork}

BioTechniques 21:237-238 (August 1996)

Density gradient centrifugation is a frequently used step to isolate cells and particles in cell biology and biochemistry. Pouring these gradients, however, can be a hassle, especially when one is inexperienced with this technique. In general, there are two different ways to achieve discrete gradients. With one technique, the densest gradient is poured first, and the other gradients of lower density are carefully overlaid. With this technique, it is of utmost importance to avoid turbulence. Thus, the tube must be turned sidewards to allow a smooth run. Unfortunately, it is still fairly difficult to avoid mixing of the two phases, especially when the density of the liquids is very close. With the other technique, the fraction of the lowest density is poured first in the tube, and the subsequent fractions of higher density are underlaid by using a Pasteur pipet or by using a device described previously (1). The problems with this approach are $(i)$ the effort needed to remove the pipet out of the medium without disturbing the gradient and (ii) the occurrence of turbulence when the liquid to be underlaid leaves the Pasteur pipet. Although the gradients are much easier to make by using a pouring device, it is still necessary to do the laborious step to set up the whole apparatus.

Here, we present a very simple and reliable method for pouring discontinuous gradients for all purposes of particle separation. The method has been tested with Percoll ${ }^{\circledR}$, Ficoll ${ }^{\circledR}$ and a number of other watery gradients (e.g., watery solutions containing different salt concentrations). The only tool necessary is a piece of a cork that may be autoclaved depending on the application. When pouring the gradients, the cork is put into the centrifuge tube and the liquids (beginning with the densest fraction) are just pipetted on top of the cork. The shape of the cork is not very important for its function, but to avoid rotating during pouring, it should look like a cuboid or a thick disc. Best results are achieved with a big piece of cork, but it must be small enough to allow for free floating on the surface of the liquid.

The principle of action is based on

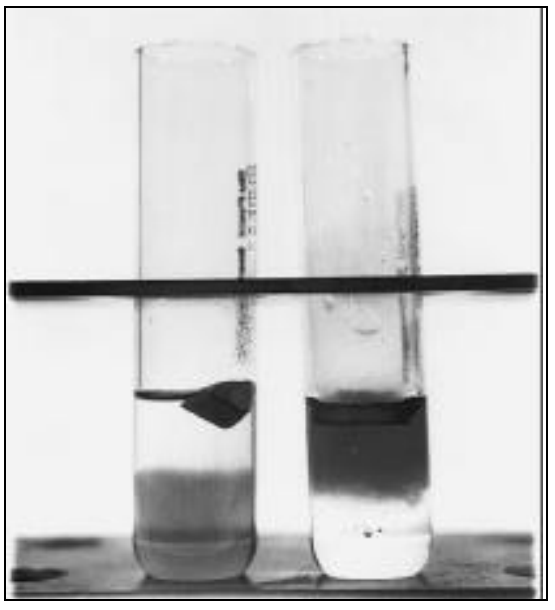

Figure 1. Discrete gradients that were poured by using a cork. The lower phases consist of $10^{\circ} \mathrm{C}$ cold water that were overlaid with $70^{\circ} \mathrm{C}$ warm water. To visualize the phases, small amounts of Coomassie ${ }^{\circledR}$ Blue were added to the cold water (left) or to the warm water (right). 
two features of the gadget. First, it disperses the kinetic energy of liquid drops dripping down because the cork acts as a suspension when floating on the surface of the gradient, which is already in the tube. Second, the cork seems to keep the liquid stream close to its surface so that the gradient poured in flows smoothly around the cork. These together are apparently very effective in avoiding turbulence between the two phases to be formed and, as shown in Figure 1, even water of different temperatures can be poured into one tube without mixing.

An exclusive application of this technique is the swim-up method for sperm (3) that requires the preparation of two phases with almost the same density. This method is used to separate cell particles and dead sperm from live ones (live sperm are motile and therefore able to enter the upper phase). In our case, two phases had to be formed in one tube without mixing. They consisted of the rabbit ejaculate and an isotonic $\mathrm{NaCl}$ solution.

Routinely, we used density gradient centrifugation in combination with this pouring technique to isolate pure zona pellucidae from ovary cell debris (2). By pouring the gradients according to the method described, the procedure was facilitated considerably since no more devices were necessary other than the cork itself. Therefore, several tubes could be handled easily in parallel. In summary, this is not only a very simple method, but it also allows for combining two liquids of almost the same density without mixing when other lowcost techniques fail.

\section{REFERENCES}

1.Feinberg, B.B., S. Berman and J. Politch. 1995. Rapid preparation of discontinuous Percoll density gradients. BioTechniques 18:802803.
2.Jansen, S., M. Quigley, W. Reik and R. Jones. 1995. Analysis of polysulfate-binding domains in porcine proacrosin, a putative zona adhesion protein from mammalian spermatozoa. Int. J. Dev. Biol. 39:501-510.

3.Lopata, A., M.J. Patullo, A. Chang and B. James. 1976. A method for collecting motile spermatozoa from human semen. Fertil. Steril. 27:677-684.

Address correspondence to Stephan Jansen, Tierärztliches Institut der Universität Göttingen, Groner Landstr. 2, 37073 Göttingen, Germany.

Received 4 December 1995; accepted 29 January 1996.

\section{Stephan Jansen, Bernd Kriegesmann and Bertram Brenig} Universität Göttingen Göttingen, Germany 